CONVOCATORIA semilleros 2020

Título del proyecto

\title{
ASPECTOS JURÍDICOS DEL CONTRATO DE GESTACIÓN SUSTITUTIVA
}

\section{Campo de acción}

Derecho Civil, personas y familia.
Transdisciplinariedad - Aporte al PIM

3. Proyección social e investigación pertinentes. Focalizar y articular la investigación y la proyección social USTA Colombia.

\section{Articulación con funciones sustantivas y el sector social y productivo} No aplica

\section{Grupo de investigación}

Estudios en derecho privado
Línea de investigación en la que se inscribe el proyecto Semillero de investigación Cuestiones del Derecho Civil y Familia

\begin{tabular}{|c|c|c|c|}
\hline Nombre del Investigador principal & Enlace CvLAC & Enlace ORCID & Enlace Google Académico \\
\hline Celmira González de Sánchez & $\begin{array}{l}\frac{\text { http://scienti.colcienci }}{\text { as.gov.co:8081/cvlac/ }} \\
\frac{\text { visualizador/generarC }}{\text { urriculoCv.do?cod rh }} \\
=0001648648\end{array}$ & $\frac{\text { https://orcid.org/0000- }}{\underline{0002-4291-1991}}$ & $\begin{array}{l}\frac{\text { https://scholar.google.es/citations?hl=es\&user=U2tg }}{\text { MxQAAAAJ\&view op=list works\&gmla=AJsN- }} \\
\frac{\text { F5Rnw VJ5q22I5Jc1Jc1JfCPEV3XR- }}{\text { 9EbK tAuc1EtNVmZIIN3q4XLP2JHLrsBgeG3ea vT }} \\
\text { qCYWCDHgp0HJSIPVxrnkXMkEg }\end{array}$ \\
\hline División & Facultad & Programa & Grupo de investigación \\
\hline Ciencias Jurídicas y políticas & Derecho & Derecho & $\begin{array}{l}\text { Estudios en derecho privado } \\
\text { Semillero de investigación Cuestiones del Derecho } \\
\text { Civil y Familia }\end{array}$ \\
\hline Nombre del Co-investigador & Enlace CvLAC & Enlace ORCID & Enlace Google Académico \\
\hline Tulia Marina Rojas Maldonado & $\begin{array}{l}\text { https://scienti.colciencia } \\
\begin{array}{l}\text { S.gov.co/cvlac/visualiza } \\
\text { dor/generarCurriculoCv. }\end{array} \\
\text { do?cod_rh }=0001724036 \\
\end{array}$ & $\frac{\underline{\text { htps://orcid.org/0000-0003- }}}{\underline{2069-6132}}$ & \\
\hline División & Facultad & Programa & Grupo de investigación \\
\hline
\end{tabular}


Ciencias Jurídicas y políticas

INVESTIGADORES PARA EL PROYECTO
Derecho

DOCENTES:

CELMIRA GONZALEZ DE SANCHEZ

MARINA ROJAS

MALDONADO
Derecho

\section{ESTUDIANTES:}

ANGEL ANDRES CARDENAS

ANDRES DARIO OTALVARO JUAN SEBASTIAN LUGO
Estudios en derecho privado - Semillero de investigación Cuestiones del Derecho Civil y Familia

\section{EL CONTRATO DE GESTACION SUSTITUTIVA}

\section{Resumen de la propuesta}

La maternidad subrogada es una de las nuevas tendencias estructurales de la familia, y en el presente artículo se hará un análisis de las instituciones del derecho civil y familia que rodena dicho tema, que serán la base jurídica para establecer una propuesta de regulación del tema, el contrato de maternidad subrogada, como debería ser abordado y sus efectos en el derecho civil y de familia.
Palabras clave

Contrato de maternidad subrogada,

TRHA (técnicas de reproducción humana asistida)

Filiación y parentesco

Contratos civiles

Responsabilidad civil

\section{Problema de investigación}

La maternidad subrogada o mal llamada alquiler de vientres, es una de las nuevas tendencias estructurales creadoras de la célula fundamental de la familia, originada en los avances médicos y científicos que se originaron a mediados del siglo XX. Dicha práctica, hace parte de las denominadas Técnicas de Reproducción Humana Asistida (TRHA), que comprenden prácticas como la inseminación artificial, la fecundación in vitro y la maternidad subrogada. Se entienden estas como herramientas que la ciencia ha brindado para hacer frente a los altos índices de infertilidad que se presentan hoy día, así como una alternativa de a la procreación por parte de parejas del mismo sexo, constituyendo una opción para superar las deficiencias médicas y las imposibilidades físicas, que imposibilitan a las parejas el poder tener hijos.

Frente al rápido avance de las ciencias el entorno jurídico se ha visto envuelto en una encrucijada, toda vez que la alta demanda que ha ido en aumento, por la necesidad de estos procedimientos, especialmente la maternidad subrogada, ha repercutido en un gran sin número de
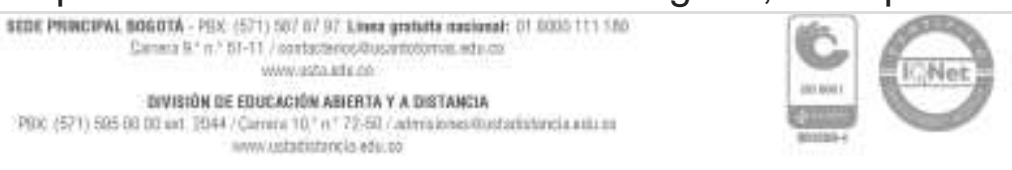
conflictos tanto de orden legal, como de orden social. Algunos países como India, Canadá, Portugal, Reino Unido, Rusia, Holanda entre otros, se han apersonado de dicho conflicto permitiendo, regulando y estableciendo los lineamientos para realizar dicha práctica. En otros como Alemania, Francia y España, dicha práctica se encuentra expresamente prohibida en los ordenamientos penales.

En el ordenamiento jurídico colombiano no está prohibida, pero tampoco se encuentra regulada. en el presente proyecto se aborda el tema de la maternidad subrogada -alquiler de vientres- a partir del estudio del mismo, desde las nociones fundamentales para el entendimiento de este como un contrato, cuya naturaleza es atípica, toda vez que no se encuentra expresamente consagrado en la legislación nacional, abordaremos los conceptos relativos a la gestación sustitutiva, desde el derecho de familia, las instituciones de la filiación, el parentesco, los derechos del menor y los conceptos relacionados con las técnicas de reproducción humana asistida (TRHA), para de manera siguiente establecer una propuesta académica sobre la estructura de la maternidad subrogada como un contrato de naturaleza típica, sus implicaciones desde el ámbito del derecho de los contratos, la responsabilidad civil y el derecho de familia.

\section{Pregunta de investigación}

¿Al entender la maternidad subrogada como un contrato regulado, cuál sería la naturaleza jurídica de este, y que implicaciones tendría para el derecho civil y de familia?

\section{Justificación}

La maternidad subrogada, al no tener regulación normativa ni a favor ni en contra en el país colombiano, ha sido la causa de varios problemas sin respuesta jurídica. Por ejemplo, el caso de la filiación entre los padres que toman el servicio y el hijo que deriva del mismo, pues teniendo en cuenta la normatividad colombiana que expone que se presume madre la que en el parto dé a luz a su hijo, sería madre la que presta el servicio de maternidad subrogada.

O ¿Qué pasa cuando no se cumple el fin por el cual se contrató a la madre gestante?, estos y más son los problemas que por falta de regulación no tienen respuesta, y que día a día van aumentando, pues la maternidad subrogada ya se vuelto una TRHA de uso común entre la sociedad.

Sin embargo regularla no es tarea fácil, pues supone la creación de una figura jurídica que será de transcendencia, por lo que se deberá tratar de cubrir cada vacío que pueda encontrarse, en cuanto, debe ser o no un contrato oneroso, cual es la responsabilidad que acarrearía el no cumplimiento del mismo y cuál es el límite en su contenido. Razón por la cual el presente proyecto determinará la naturaleza jurídica del contrato de maternidad subrogada, sus efectos en el derecho civil y de familia; con el fin de entregar una propuesta jurídica que se adapte a la realidad del país colombiano y que elimine la mayor cantidad de vacíos normativos que se puedan presentar en su regulación
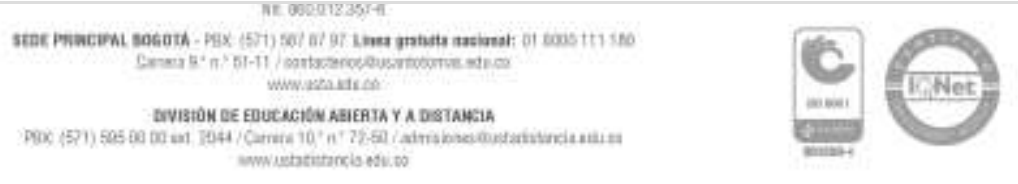


\section{Objetivo general}

Determinar la naturaleza jurídica del contrato de maternidad subrogada y sus efectos en el derecho civil y de familia.

\section{Objetivos específicos}

1. Establecer los conceptos básicos sobre TRHA, maternidad subrogada, derecho de familia y menores, contratos civiles y comerciales

2. Analizar en el sistema jurídico, las formas de los contratos y las relaciones de familia en las TRHA

3. Analizar sistemas jurídicos extranjeros a fin de presentar desde el derecho comparado, la posición de algunos estados frente al tema.

4. determinar cuáles serían los efectos jurídicos en el derecho civil y de familia al regular el contrato de maternidad subrogada.

5. Diseñar una propuesta jurídica de regulación del contrato de maternidad subrogada.

\section{Metodología}

De acuerdo a la investigación planteada, en el presente estudio se utilizara un método consultivo, en el que se ahondara en la búsqueda jurídica y doctrinal de las instituciones que componen el proyecto, todas relacionadas con el derecho civil y de familia, descriptivo al analizar y describir una problemática contemporánea, comparativo en cuanto se realizaran análisis comparativos con derecho extranjero a fin de presentar comparativos, por ultimo propositivo ya que se presentara una propuesta de regulación del tema, específicamente en el derecho civil y familia, sobre permitir y regular, el contrato de maternidad subrogada.

1. Informe de investigación

\section{Resultados esperados}

2. Producción de video o contenido audiovisual con los resultados del proyecto

3. Socialización de resultados (sometido a disponibilidad de reunión presencial o virtual)

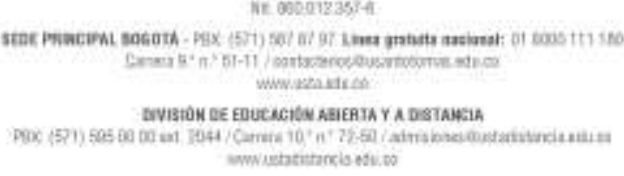




\section{Cronograma}

\begin{tabular}{|c|c|c|c|c|c|c|c|c|c|c|c|c|c|c|c|c|c|c|c|c|c|c|c|c|c|c|c|c|c|c|c|c|c|c|c|c|c|c|c|c|c|c|c|}
\hline \multirow[b]{2}{*}{ ACTIVIDADES } & \multirow{2}{*}{ RESPONSABLE } & \multicolumn{2}{|c|}{ FECHA } & \multicolumn{4}{|c|}{ FEBRERO } & \multicolumn{4}{|c|}{ MARZO } & \multicolumn{4}{|c|}{ ABRIL } & \multicolumn{4}{|c|}{ MAYO } & \multicolumn{4}{|c|}{ JUNIO } & \multicolumn{4}{|c|}{ JULIO } & \multicolumn{4}{|c|}{ AGOSTO } & \multicolumn{4}{|c|}{ SEPTIEMBRE } & \multicolumn{4}{|c|}{ OCTUBRE } & \multicolumn{4}{|c|}{ NOVIEMBRE } \\
\hline & & Inicio & Fin. & 1 & 2 & 3 & 4 & 5 & 6 & 7 & 8 & 9 & 10 & 11 & 12 & 13 & 14 & 15 & 16 & 17 & 18 & 19 & 20 & 21 & 22 & 23 & 24 & 25 & 26 & 28 & 29 & 30 & 31 & 32 & 33 & 34 & 35 & 36 & 37 & 38 & 39 & 40 & 41 \\
\hline $\begin{array}{l}\text { Planteamiento } \\
\text { del problema }\end{array}$ & $\begin{array}{l}\text { Investigador principal } \\
\text { y coinvestigador }\end{array}$ & $\begin{array}{r}01 \\
\text { feb }\end{array}$ & $01 \mathrm{mar}$ & & & & $X$ & & & & & & & & & & & & & & & & & & & & & & & & & & & & & & & & & & & & \\
\hline $\begin{array}{l}\text { Búsqueda y } \\
\text { selección de } \\
\text { informacion }\end{array}$ & $\begin{array}{l}\text { Investigador principal } \\
\text { y coinvestigador }\end{array}$ & $\begin{array}{r}01 \\
\text { feb }\end{array}$ & $30 \mathrm{abr}$ & & & & & & & & & & & & X & & & & & & & & & & & & & & & & & & & & & & & & & & & & \\
\hline $\begin{array}{l}\text { Análisis de } \\
\text { información }\end{array}$ & $\begin{array}{l}\text { Investigador principal } \\
\text { y coinvestigador }\end{array}$ & $\begin{array}{c}01 \\
\text { may }\end{array}$ & $\begin{array}{c}31 \\
\text { may }\end{array}$ & & & & & & & & & & & & & & & & $\mathrm{x}$ & & & & & & & & & & & & & & & & & & & & & & & & \\
\hline $\begin{array}{l}\text { Desarrollo de } \\
\text { objetivos }\end{array}$ & \begin{tabular}{|l|} 
Investigador principal \\
y coinvestigador
\end{tabular} & $\begin{array}{r}01 \\
\text { feb }\end{array}$ & $\begin{array}{c}31 \\
\text { may }\end{array}$ & & & & & & & & & & & & & & & & $X$ & & & & & & & & & & & & & & & & & & & & & & & & \\
\hline $\begin{array}{l}\text { Construcción } \\
\text { de texto }\end{array}$ & $\begin{array}{l}\text { Investigador principal } \\
\text { y coinvestigador }\end{array}$ & $\begin{array}{c}01 \\
\text { may }\end{array}$ & 31 oct & & & & & & & & & & & & & & & & $X$ & & & & & & & & & & & & & & & & & & & & & & & & \\
\hline $\begin{array}{l}\text { Elaboración } \\
\text { del primer } \\
\text { borrador del } \\
\text { articulo }\end{array}$ & $\begin{array}{l}\text { Investigador principal } \\
\text { y coinvestigador }\end{array}$ & $\begin{array}{c}01 \\
\text { mayo }\end{array}$ & 20 jun & & & & & & & & & & & & & & & & & & & & & $\mathrm{x}$ & & & & & & & & & & & & & & & & & & & \\
\hline $\begin{array}{l}\text { Correcciones y } \\
\text { entrega de la } \\
\text { mitad del } \\
\text { articulo }\end{array}$ & $\begin{array}{l}\text { Investigador principal } \\
\text { y coinvestigador }\end{array}$ & $\begin{array}{c}01 \\
\text { may }\end{array}$ & 30 jun & & & & & & & & & & & & & & & & & & & & & & & & & $x$ & & & & & & & & & & & & & & & \\
\hline $\begin{array}{l}\text { Desarrollo de } \\
\text { la segunda } \\
\text { mitad }\end{array}$ & $\begin{array}{l}\text { Investigador principal } \\
\text { y coinvestigador }\end{array}$ & 01 jul & 31 jul & & & & & & & & & & & & & & & & & & & & & & & & & & & & & & & & $\mathrm{X}$ & & & & & & & & \\
\hline $\begin{array}{l}\text { Construcción } \\
\text { final del articulo }\end{array}$ & $\begin{array}{l}\text { Investigador principal } \\
\text { y coinvestigador }\end{array}$ & 1 ago & 31 oct & & & & & & & & & & & & & & & & & & & & & & & & & & & & & & & & & & & & $\mathrm{X}$ & & & & \\
\hline $\begin{array}{l}\text { Ponencia o } \\
\text { evento de } \\
\text { presentación } \\
\text { de resultado }\end{array}$ & $\begin{array}{l}\text { Investigador } \\
\text { principal y } \\
\text { coinvestigador }\end{array}$ & 1 nov & 10 nov & & & & & & & & & & & & & & & & & & & & & & & & & & & & & & & & & & & & & & $\mathrm{X}$ & & \\
\hline & & & & & & & & & & & & & & & & & & & & & & & & & & & & & & & & & & & & & & & & & & & \\
\hline
\end{tabular}

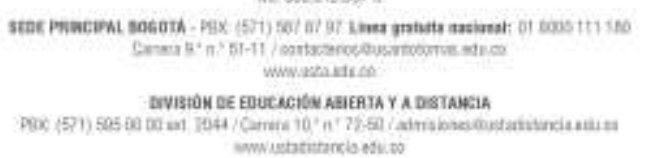




\begin{tabular}{|c|c|c|c|c|c|}
\hline FINANCIACIÓN & RECURSO & DESCRIPCIÓN & $\begin{array}{c}\text { Valor } \\
\text { partida }\end{array}$ & $\begin{array}{c}\text { Valor } \\
\text { contrapartida } \\
\text { (Externa) }\end{array}$ & Total (\$) \\
\hline \multirow{4}{*}{ RUBROS } & Servicios Técnicos & & & & $\$ 0$ \\
\hline & Salidas de campo & & & & $\$ 0$ \\
\hline & Equipos & & & & $\$ 0$ \\
\hline & $\begin{array}{l}\text { Materiales, insumos y } \\
\text { software }\end{array}$ & & & & $\$ 0$ \\
\hline \multirow{6}{*}{ BOLSAS } & Papelería & & & & $\$ 0$ \\
\hline & Fotocopias & & & & $\$ 0$ \\
\hline & Material bibliográfico & & & & $\$ 0$ \\
\hline & Auxilio de transporte & & & & $\$ 0$ \\
\hline & Movilidad & & & & $\$ 0$ \\
\hline & $\begin{array}{l}\text { Publicaciones } \\
\text { (Artículos, proceso } \\
\text { editorialy traducción) }\end{array}$ & & & & $\$ 0$ \\
\hline & & & \multicolumn{2}{|c|}{ TOTAL DEL PROYECTO: } & $\$ 0$ \\
\hline
\end{tabular}




\section{Bibliografía}

Cabanellas, G. (1986). Diccionario Enciclopedico de Derecho Usual TOMO VII (Vigesima ed., Vol. VII). Buenos Aires: Heliasta S.R.L.

Cárdenas Rojas, L. V. (2015 ). Validez y eficacia del contrato de maternidad subrogada en el ordenamiento juridico colombiano. Conflicto \& Sociedad ISSN 23226900, 3(1), 101-110.

Congreso de la Republica de Colombia . (2006 ). Ley 1098 de noviembre 8 de 2006, Por la cual se expide el Código de la Infancia y la Adolescencia . Bogotá D.C. : Congreso de la Republica de Colombia .

Cruz Palomo, J. C., \& Castro Ortiz, L. L. (2014). La omisión legislativa en materia de contrato de alquiler de vientre materno en Colombia y consecuencias jurídicas. Criterio Jurídico Garantista ISSN: 2145-3381(11), 110-125.

Duran Mantilla, J. G. (2016). Iusfilosofia con ventanas, una filosofia juridica mirada desde el punto medio aristotelico. Bogotá D.C.: Grupo editorial Ibañez.

Gonzalez de Cancino, E. (1995). Los retos juridicos de la genetica. Bogotá D.C.: Universidad Externado de Colombia, Centro de Estudios sobre Genetica y Derecho .

López De Armas, K. M., \& Amado Amado, C. (2014). Determinación de la filiación materna en Colombia en la práctica de la maternidad delegada. Revista de Derecho Privado ISSN 1909-7794(52), 3-18 .

Moreno Diaz, V., Gongora Vega, A. I., \& Duran Vinazco, R. (2017). La voluntad en la filiacion de los matrimonios y uniones homoparentales. En O. A. Ramirez Arenas, Justicia Constitucional Tomo II (Vol. Tomo II, págs. 15-38). Bogotá D.C.: Grupo Editorial Ibañez.

Profesionales por la etica. (01 de Abril de 2015). profesionalesetica.org. Obtenido de http://profesionalesetica.org: http://profesionalesetica.org/wpcontent/uploads/2015/06/V-aquiler-web.pdf

Quiroz Monsalvo, A. W. (2016). ¿Que es la personalidad juridica para el derecho? En O. Woolcott Oyague, \& L. Vargas Espitia, Problemas actuales del derecho privado y derecho laboral (págs. 13-25). Bogotá D.C.: Grupo Editorial Ibañez.

Romero Rubio, C. A. (2019). Maternidad subrogada: lagunas en el ordenamiento jurídico colombiano. ¿Con qué elementos cuenta el juez para adoptar su decisión? Revista Iusta ISSN: 1900-0448(50), 175-189.

Suarez Parada, A. L. (2008). Reproducción Humana Asistida y Filiacion en el Derecho de Familia Colombiano. Revista Via Inveniendi et Iudicandi ISSN: 19090528(6), Version digital no numerada. Obtenido de https://2019-vlex-com.crai-ustadigital.usantotomas.edu.co/\#vid/42184963

Valencia Zea, A. (1998). Derecho civil, Tomo V, Derecho de familia (Sexta ed.). Bogotá D.C.: Temis S.A. .

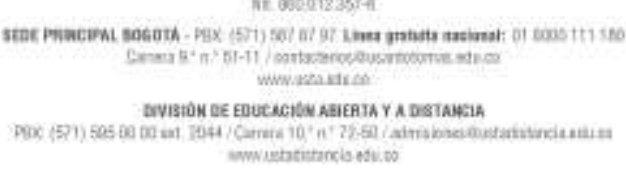

